\title{
An Overview of Two-level Supersaturated Designs with Cyclic Structure
}

Stelios D. Georgiou, Department of Statistics and Actuarial-Financial Mathematics, University of the Aegean, Samos, Greece. Email: stgeorgiou@aegean.gr

Danel Draguljić, Department of Statistics, The Ohio State University, Columbus, OH 43210,USA.Email: danel@stat.osu.edu

Angela M. Dean, Department of Statistics, The Ohio State University, Columbus, OH 43210, USA. Email: dean.9@osu.edu

Received: January 22, 2008 Revised: October 27, 2008

\section{Abstract}

An overview is given of the link between the $k$-circulant method of construction of two-level supersaturated designs and construction methods based on cyclic incomplete block designs. It is shown that this link enables a simple formula for the $E s^{2}$-efficiency of all such designs to be derived. Generators are given for $E s^{2}$-optimal and near-optimal designs that extend the range of previously known designs or that have a smaller number of highly correlated column pairs.

AMS Subject Classification: Primary: 62K15. Secondary: 62K05.

Key-words: Circulant design; Cyclic design; Efficiency; $E s^{2}$.

\section{Introduction}

The goal of a screening experiment is to identify the few most "active" factors from a very large number of potentially important factors. Supersaturated designs have been advocated for screening when relatively few observations can be taken and when it can be assumed that the factor main effects will be much larger than the interactions. Some supersaturated designs allow interactions to be investigated but, usually, main effect models are fitted.

Various methods of analysis of supersaturated designs have been investigated in the literature. Frequentist methods include forward selection and stepwise regression (Westfall, Young and Lin, 1998; Abraham, Chipman and Vijayan, 1999; Kelly and Voelkel, (2000), penalized least squares methods (Lin, 1995; Li and Lin, 2002), the Dantzig selector (Phoa, $* 1559-8608 / 09-2 / \$ 5+\$ 1 p p-$ see inside front cover 
Pan and $\mathrm{Xu}, 2007)$. Bayesian methods that can be used for analysing supersaturated designs have been investigated by Chipman, Hamada and Wu (1997); Beattie, Fong and Lin, (2002) Meyer and Wilkinson, (1998), among others.

In trying to extract information about a large number of factor main effects from very few observations, it is inevitable that the chance of mis-identifying the most important effects can be fairly high. This is due to the fact that main effect estimates are correlated and sometimes more than one model fits the data equally well. To minimize mistakes, it is advisible to use designs with main effect contrast correlations as small as possible. Chen and Lin (1998) and Liu, Ruan and Dean (2006) suggest that a maximum correlation of 0.333 is desirable, but this cannot always be achieved for very large numbers of factors. However, provided that there are very few active factors (factor sparsity, see Box and Meyer, 1986) and provided that important effects are very large, it appears possible to identify most of the active factors, in general.

Supersaturated designs were introduced by Satterthwaite (1959) in terms of random balance designs. The first systematic study of the construction of efficient supersaturated designs was given by Booth and Cox (1962) who defined several criteria, including minimization of $E s^{2}$ (or ave $\left(s^{2}\right)$ ) and minimization of maximum column correlation, for evaluating and comparing designs. In recent years, the construction and analysis of supersaturated designs has been an especially active area of research. Construction methods based on Hadamard matrices have been investigated, for example, by Lin (1993), Wu (1993) Tang and Wu (1997), Cheng (1997), Butler, Mead, Eskridge and Gilmour (2001); methods based on balanced incomplete block designs have been discussed by Nguyen (1996), Cheng (1997), Bulutoglu and Cheng (2004); computer algorithmic methods have been employed by Lin (1995), Nguyen (1996), Li and Wu, (1997), and Bulutoglu and Cheng (2004); cyclic methods of construction have been explored, for example, by Liu and Zhang (2000), Eskridge, Gilmour, Mead, Butler and Travnicek (2004), Liu and Dean (2004), and Georgiou (2008). All of the designs listed in the above papers are optimal or highly efficient under the $E s^{2}$ criterion. Cheng (1997), Butler, Mead, Eskridge and Gilmour (2001), and Bulutoglu and Cheng (2004) give fairly complete solutions to a wide range of $E s^{2}$-optimal supersaturated designs with number of runs $n$ a multiple of 4 and also $n=10,14,18$ for a wide range of numbers of factors $m$.

In this paper, we concentrate on the link between cyclic methods of construction of $n$ run supersaturated designs with $m=k(n-1)$ two-level factors (for any specified integer $k$ ). The method described by Nguyen (1996) for construction of $E s^{2}$-optimal supersaturated designs was to adjoin the incidence matrices of pairs of cyclic balanced incomplete block designs (BIBD). An extension of this method was discussed by Eskridge, Gilmour, Mead, Bultler and Travnicek (2004), where the incidence matrices of regular graph designs as well as balanced incomplete block designs were used. Liu and Zhang (2000) developed an algorithm for constructing $E s^{2}$-optimal supersaturated designs from cyclic BIBDs. A related method based on cyclic generators was given by Liu and Dean (2004). The link between these construction methods is explored in Section 3 and is exploited in Section 4 to give a simple formula for the $E s^{2}$-efficiency of all such designs. Cyclic generators are given for $E s^{2}$-optimal and near-optimal designs that extend the range of previously known designs, or that have a smaller number of highly correlated pairs of columns.

Since this paper concentrates on cyclic generation of supersaturated designs, it does not cover all the known $E s^{2}$-optimal designs. In particular $E s^{2}$-optimal designs based on non- 
cyclic Hadamard matrices and incomplete block designs are not covered. Various non-cyclic solutions for $E s^{2}$-optimal supersaturated designs for ranges of $n$ and $m$ have been provided by Butler, Mead, Eskridge and Gilmour (2001), Bulutoglu and Cheng (2004), Ryan and Bulutoglu (2007) and Das, Dey, Chan and Chatterjee (2008).

\section{Bounds for $E s^{2}$-efficiency}

We represent a supersaturated design $d$ with two-level factors by a "design matrix" or "treatment matrix", $T$, whose rows represent the $n$ treatment combinations to be observed and whose columns designate the $m$ factors to be examined. The $(i, f)$ th element of $T$ determines the level at which factor $f$ is to be observed in the $i$ th observation or "run". We code the high and low levels of each factor as +1 and -1 in $T$, and abbreviate these to "+" and "-" where convenient. For mean-orthogonal two-level designs, where the levels of each factor are observed the same number of times, the columns of $T$ coincide with main effect contrasts. We will use the standard main effects model

$$
Y=X \phi+\varepsilon
$$

where $X=\left[\mathbf{1}_{n} T\right]$ and $\mathbf{1}_{n}$ denotes the $n$-dimensional column vector with all entries equal to $1 ; \phi$ is a vector containing the mean and main effect parameters and $\varepsilon$ is a vector of $n$ independent and identically distributed normal random variables with mean zero and variance $\sigma^{2}$. The information matrix for estimating $\phi$ is

$$
\left[\begin{array}{cc}
n & 0 \\
0 & T^{\prime} T
\end{array}\right]
$$

Let $s_{i j}$ be the element in the $i$ th row and $j$ th column of $T^{\prime} T(i, j=1, \ldots, m)$. Then, $s_{i j}=t_{i}^{\prime} t_{j}$, where $t_{i}$ and $t_{j}$ are, respectively, the columns $i$ and $j$ of $T$. If $s_{i j}=0$, the $i$ th and $j$ th main effect contrasts are orthogonal and can be estimated independently. If $n$ is even, but not a multiple of 4 , then $s_{i j}$ cannot equal 0 and the main effect contrasts for factors $i$ and $j$ will be said to be nearly orthogonal if $s_{i j}$ is close to 0 (that is, $s_{i j}= \pm 2$ when $n \equiv 2(\bmod 4)$ ). When $s_{i j}= \pm n$ then the main effects of factors $i$ and $j$ are completely aliased, and such designs are usually avoided.

Booth and Cox (1962) proposed as a criterion for comparing designs the minimization of the average correlation between the main effect contrasts, called ave $\left(s^{2}\right)$, or $E s^{2}$, where

$$
E s^{2}=\sum_{1 \leq i<j \leq m} s_{i j}^{2} /\left(\begin{array}{c}
m \\
2
\end{array}\right) .
$$

A supersaturated design is said to be $E s^{2}$-optimal if no other design of the same size has higher $E s^{2}$-efficiency, defined as LB $/ E s^{2}$, where LB is an appropriate lower bound for $E s^{2}$ as discussed below. Although minimizing $E s^{2}$ (which is a function of the entries of $T^{\prime} T$ ) is not identical to the criterion of minimizing the average correlation between the contrast estimators (which is a function of the entries of $\left[X^{\prime} X\right]^{-1}$ ), it does lead to highly efficient designs under this latter criterion provided that the designs are mean-orthogonal.

Lower bounds for $E s^{2}$ for supersaturated designs with $n$ runs and $m=k(n-1)$ factors 
have been derived for $n=0$ mod 4 by Nguyen (1996) and Tang and Wu (1997), and bounds for other values of $n$ and $m$ have been given by Cheng (1997), Butler, Mead, Estridge and Gilmour (2001), and Das, Dey, Chan and Chatterjee (2008). Using the results of these papers for the designs discussed here, with $m=k(n-1)$ factors $(k>1)$, we have the following lower bounds for $E s^{2}$ :

(i) Nguyen (1996), Tang and Wu (1997), Cheng (1997).

If $n \equiv 0(\bmod 4)$ with any $k>1$ or if $n \equiv 2(\bmod 4)$ with $k$ even, then

$$
E s^{2} \geq L B=\frac{m-n+1}{(m-1)(n-1)} n^{2} .
$$

(ii) Das et al. (2008).

If $n \equiv 2(\bmod 4)$ with $k$ odd, then

$$
E s^{2} \geq \max \{L B, 4\}, \quad \text { where } L B=\frac{n^{2}(m-n+1)}{(m-1)(n-1)}+\frac{2 n^{2}-4 n+x}{m(m-1)}
$$

and $x=32$ if $\left\{\frac{m-1-2 i}{4}+\left[\frac{m+(1+2 i)(n-1)}{4(n-1)}\right]\right\} \equiv(1-i)(\bmod 2)$ for $i=0$ or 1 , and $[\cdot]$ denotes integer part. Otherwise, $x=0$. It can be shown that, for $k \geq 3, L B>4$ so that the bound is given by (2.3).

\section{Cyclic construction methods}

\subsection{Cyclic generators}

The cyclic construction method used by Plackett and Burman (1946) for orthogonal saturated designs was generalized by Liu and Dean (2004) to obtain a class of supersaturated designs called $k$-circulant designs. A $k$-circulant design with $n=2 t$ runs (for $t$ an integer) and $m=k(n-1)$ factors each having two levels is constructed by cycling elements of a generator $G=\left(g_{1}, \ldots, g_{m}\right)$ containing $k t$ elements equal to -1 and $k(t-1)$ elements equal to +1 , where the cycling is done $k$ elements at a time. A row of +1 's is added to the resulting $(n-1) \times m$ array $D$ to give the supersaturated design matrix $T=\left[D^{\prime}, \mathbf{1}_{k(n-1)}\right]^{\prime}$. The generators listed by Liu and Dean (2004) give rise to designs that are mean-orthogonal and which are either $E s^{2}$-optimal or have at least $96.4 \%$ efficiency. The following three results summarize the links between $k$-circulant designs, the concatenation of $k$ individual saturated cyclic (1-circulant) designs and the designs of Nguyen (1996), Eskridge, Gilmour, Mead, Butler and Travnicek (2004), and Bulutoglu and Cheng (2004) formed from the incidence matrices of cyclic incomplete block designs. These links are illustrated in Example 3.1.

Result 3.1 (Liu and Dean, 2004, Section 3). Any k-circulant mean-orthogonal supersaturated design $T=\left[D^{\prime}, \mathbf{1}\right]^{\prime}$ with $n=2 t$ runs, $m=k(n-1)$ factors and generator $G=\left(g_{1}, g_{2}, \ldots, g_{m}\right)$ is equivalent to the concatenation of $k$ individual 1-circulant designs, $T_{1}, T_{2}, \ldots, T_{k}$, each having $(n-1)$ factors, where the ith design $T_{i}=\left[D_{i}^{\prime}, \mathbf{1}_{n-1}\right]^{\prime}$ is obtained by cycling the elements of the generator $G_{i}=\left(g_{i}, g_{i+k}, g_{i+2 k}, \ldots, g_{i+(n-2) k}\right)$ and adding $a$ row of 1 's, $(i=1, \ldots, k)$. 
Result 3.1, together with expression (2) of Eskridge, Gilmour, Mead, Butler and Travnicek (2001) leads to the following result.

Result 3.2. Any $k$-circulant supersaturated design matrix $T=\left[D^{\prime}, \mathbf{1}_{k(n-1)}\right]^{\prime}$ with $n=2 t$ runs and $m=k(n-1)$ factors and generator $G=\left(g_{1}, g_{2}, \ldots, g_{m}\right)$ can be obtained from the incidence matrix, $N=\left[N_{1}, \ldots, N_{k}\right]$ of an incomplete block design consisting of $k$ cyclic subdesigns with $n-1$ blocks each, where the ith subdesign $(1 \leq i \leq k)$ has circulant incidence matrix $N_{i}$ with first row

$$
2^{-1}\left(1+g_{i}, 1+g_{i+k}, 1+g_{i+2 k}, \ldots 1+g_{i+(n-2) k}\right) .
$$

Then $D=2 N-\mathbf{1}_{n-1} \mathbf{1}_{m}^{\prime}$.

The third result links $E s^{2}$-optimality of certain $k$-circulant designs and the existence of balanced incomplete block designs.

Result 3.3 (Cheng, 1997, Section 2). The incomplete block design in Result 3.2 with incidence matrix $N$ is a balanced incomplete block design if and only if the corresponding $k$-circulant design $T$ is $E s^{2}$-optimal attaining bound (2.2).

Example 3.1. Consider the 3 -circulant $E s^{2}$-optimal supersaturated design with $n=12=2 t$ $(t=6)$ experimental runs and $m=33=(2 t-1) k$ factors listed in Table 4 of Liu and Dean (2004). The listed generator has $k t=18$ elements equal to -1 and $k(t-1)=15$ elements equal to 1 , as follows

$$
G=(-----------+++---++++-+-+--++++++) .
$$

Cycling $k=3$ elements at a time gives an $E s^{2}$-optimal 3-circulant supersaturated design with maximum column correlation $r_{\max }=\left(\max \left|s_{i j}\right|\right) / n=0.67$ and $E s^{2}=9$. If the columns of the design are rearranged into the following order:

$$
1,4,7, \ldots, 31 ; 2,5, \ldots, 32 ; 3,6, \ldots, 33 \text {; }
$$

the design can be seen to be the concatenation of $k=3$ separate cyclic (1-circulant) designs with respective generators

$$
\begin{aligned}
& G_{1}=(----+-+-+++) \\
& G_{2}=(----+-++-++) \\
& G_{3}=(---+-++--++)
\end{aligned}
$$

where $G_{i}$ consists of elements $i, i+3, i+6, \ldots, i+30$ of $G$. The generators can further be rewritten by noting the positions which hold +1 , that is the positions in the first rows of the cyclic subarrays that hold +1 :

$$
G_{1}^{(R)}=(5,7,9,10,11) ; \quad G_{2}^{(R)}=(5,7,8,10,11) ; \quad G_{3}^{(R)}=(4,6,7,10,11) .
$$

Arrays that are formed by cycling the rows may also be obtained by cycling the columns, so we may rewrite the $i$ th generator in terms of the $(t-1)$ positions $p_{i 1}, \ldots, p_{i, t-1}$ of the first column of array $i$ which contain +1 . Then, $p_{i j}=(n+1)-a_{i j}$, where $a_{i j}$ is the $j t h$ column 
entry in $G_{i}^{(R)}$. Thus, with $n=12$, the three generators become

$$
G_{1}^{(C)}=(8,6,4,3,2) ; \quad G_{2}^{(C)}=(8,6,5,3,2) ; \quad G_{3}^{(C)}=(9,7,6,3,2) .
$$

In this form the generators can be regarded as the initial blocks of cyclic subdesigns with $v=11$ treatments (coded $1,2, \ldots, 11)$ and $b=11$ blocks of size 5 . This is the notation used by Liu and Zhang (2000), Butler, Mead, Eskridge and Gilmour (2001), and Eskridge, Gilmour, Mead, Butler and Travnicek (2004), after subtracting 1 from each entry. It can be verified that the three subdesigns, taken together, form a balanced incomplete block design with 33 blocks of size 5 .

\subsection{The autocorrelation function}

Liu and Dean (2004, Theorem 3.2) showed that the elements $s_{i j}$ of $T^{\prime} T$ can be written in terms of the elements of the generators of the $k$-circulant design $T$. In Lemma 3.1, we express this result in terms of the periodic autocorrelation functions, defined below, which are used in Section 4 for the construction of $E s^{2}$-optimal and near optimal designs.

Let $A$ be a (finite) sequence of $\ell$ real numbers $\left\{a_{0}, a_{1}, \ldots, a_{\ell-1}\right\}$. The periodic autocorrelation function $P_{A}(x)$ is defined by

$$
\begin{aligned}
& P_{A}(x)=\sum_{q=0}^{\ell-1} a_{q} a_{q+x}, \quad x=0,1, \ldots,[\ell / 2] \\
& P_{A}(x)=P_{A}(\ell-x)
\end{aligned}
$$

where $q+x$ is reduced modulo $\ell$ and $[z]$ is the integer part of $z$.

Lemma 3.1. Let $T$ be a design matrix of the form $T=\left[D^{\prime}, \mathbf{1}\right]^{\prime}$, where $D$ is a 1-circulant matrix with generator $G=\left(g_{1}, g_{2}, \ldots, g_{n-1}\right)$. Then, the $(i, j)$ th element $s_{i j}$ of the matrix $T^{\prime} T$ can be expressed in terms of the generator elements as

$$
s_{i j}=1+\sum_{q=0}^{n-2} g_{q+i} g_{q+j}=1+\sum_{q=0}^{n-2} g_{q} g_{q+|i-j|}=1+P_{G}(|i-j|), \quad i, j=1,2, \ldots, n-1,
$$

where $g_{0}$ is defined to be $g_{n-1}$. Also,

where, if $q+x>n-1$ then it is replaced by $q+x-(n-1)$.

In Theorem 3.1, we generate a $k$-circulant supersaturated design by concatenating $k 1$ circulant designs and show how the value of $s_{i j}$ can be calculated by using the generators of the $k$ individual 1-circulant design matrices.

Theorem 3.1. Suppose that $D_{z}$ is a 1-circulant matrix with first row $G_{z}=\left(g_{1}^{(z)}, g_{2}^{(z)}, \ldots, g_{n-1}^{(z)}\right)$, $g_{j}^{(z)} \in\{1,-1\}, z=1,2, \ldots, k, j=1,2, \ldots, n-1$ and $n=2 t, t>0$. Denote by $T$, the $k$ - 
circulant mean-orthogonal supersaturated design defined by

$$
T=\left[\begin{array}{c}
D \\
\mathbf{1}_{k(n-1)}^{\prime}
\end{array}\right]=\left[\begin{array}{cccc}
D_{1} & D_{2} & \cdots & D_{k} \\
\mathbf{1}_{n-1}^{\prime} & \mathbf{1}_{n-1}^{\prime} & \cdots & \mathbf{1}_{n-1}^{\prime}
\end{array}\right] .
$$

The $(i, j)$ th element $1 \leq i, j \leq k(n-1)$ of $T^{\prime} T$ can be expressed in terms of the generator elements as

$$
s_{i j}=1+\sum_{u=0}^{n-2} g_{u+a}^{(z)} g_{u+b}^{(w)}
$$

where $z, w$ are the unique integers such that $1 \leq z, w \leq k$ and $(z-1)(n-1)<i \leq z(n-1)$, $(w-1)(n-1)<j \leq w(n-1)$, and where $a=i-(z-1)(n-1)$ and $b=j-(w-1)(n-1)$.

Proof. The proof follows from Lemma 3.1.

Since any $p$-circulant supersaturated design is equivalent to concatenating $p$ 1-circulant designs, Theorem 3.1 can be extended by replacing any subset(s) of $p$ 1-circulant matrices $D_{i}$ by a corresponding $p$-circulant matrix. The following Theorem 3.2 expresses the periodic autocorrelation function of a $k$-circulant supersaturated design in terms of the $s_{i j}$ 's (offdiagonal elements of $T^{\prime} T$ ).

Theorem 3.2. Let $T$ be an $n \times m k$-circulant mean-orthogonal supersaturated design with generator $G=\left\{g_{1}, g_{2}, \ldots, g_{k}, g_{k+1}, \ldots, g_{2 k}, \ldots, g_{m-k+1}, \ldots, g_{m}\right\}$ and $m=k(n-1), n=2 t$, $t>0$. Then

$$
P_{G}(x)=\sum_{j=1}^{k} s_{j, j+x}-k, \quad x=0,1, \ldots,[m / 2] .
$$

Proof. Define $g_{0}=g_{m}$, then

$$
\begin{aligned}
P_{G}(x) & =\sum_{i=1}^{m} g_{i} g_{i+x} \\
& =\sum_{q=0}^{n-2} g_{q k+1} g_{q k+1+x}+\sum_{q=0}^{n-2} g_{q k+2} g_{q k+2+x}+\ldots+\sum_{q=0}^{n-2} g_{q k+k)} g_{q k+k+x} \\
& =\sum_{j=1}^{k}\left(\sum_{q=0}^{n-2} g_{q k+j} g_{q k+j+x}+1\right)-k \\
& =\sum_{j=1}^{k} s_{j, j+x}-k
\end{aligned}
$$

where the last line follows from the fact that $s_{q k+j, q k+j+x}=s_{j, j+x}$, for all $q=0,1, \ldots, n-2$, and where $k(n-1)$ is subtracted from any subscript greater than $k(n-1)$.

In Theorem 3.3, we show how the elements of $T^{\prime} T$ for a mean-orthogonal supersaturated design $T$ are linked to the elements of the concurrence matrix of the dual of the corresponding incomplete block design. Then for the special case of $k$-circulant mean-orthogonal supersaturated designs, Theorem 3.4 shows the link between the elements of $T^{\prime} T$ and the 
elements of the concurrence matrix of the corresponding incomplete block design. In the theorem, the notation $J_{a, b}$ denotes an $a \times b$ matrix of unit elements. If $a=b$, we write $J_{a}$.

Theorem 3.3. Let $N$ be the incidence matrix of the incomplete block design d corresponding to supersaturated design $T=\left[D^{\prime}, \mathbf{1}_{k(n-1)}\right]^{\prime}$ so that $N=\left[D+J_{(n-1), k(n-1)}\right] / 2$. Let $\mu_{p q}$ be the $(p, q)$ th element of the concurrence matrix of the dual of $d$. Then

$$
s_{p q}=4 \mu_{p q}-(n-4) .
$$

Proof. If $N$ is the incidence matrix of $d$, then $N^{\prime}$ is the incidence matrix and $N^{\prime} N$ is the concurrence matrix of the dual of $d$ (see Street and Street, 1987, Section 2.2). Further

$$
\begin{aligned}
4 N^{\prime} N & =D^{\prime} D+D^{\prime} J_{(n-1), k(n-1)}+J_{k(n-1),(n-1)} D+J_{k(n-1),(n-1)} J_{(n-1), k(n-1)} \\
& =D^{\prime} D-J_{k(n-1)}-J_{k(n-1)}+(n-1) J_{k(n-1)} \\
& =D^{\prime} D+(n-3) J_{k(n-1)} .
\end{aligned}
$$

Since $s_{p q}$ is the $(p, q)$ th element of $T^{\prime} T=D^{\prime} D+J_{k(n-1)}$, itfollows that

$$
4 \mu_{p q}=\left(s_{p q}-1\right)+(n-3),
$$

and the result follows.

Theorem 3.4. Let $\lambda_{p q}$ be the $(p, q)$ th element of the concurrence matrix $N N^{\prime}$ of incomplete block design d with incidence matrix $N=\left[N_{1}, N_{2}, \ldots, N_{k}\right]$, where $N_{i}=\left[D_{i}+J_{n-1}\right] / 2$, corresponding to $k$-circulant supersaturated design $T$ as given in (3.2). Then

$$
4 \lambda_{p, q}=\left[\sum_{z=1}^{k} s_{n-p, n-q}^{(z)}\right]+k(n-4),
$$

where $s_{n-p, n-q}^{(z)}$ is element $[(n-p),(n-q)]$ of $\left(D_{z}^{\prime} D_{z}+J_{n-1}\right), z=1, \ldots, k$.

Proof. Let $T$ be as given in (3.2) and let $N=\left[D+J_{n-1, k(n-1)}\right] / 2$ be the incidence matrix of the corresponding incomplete block design. Then,

$$
\begin{aligned}
4 N N^{\prime} & =\left[D+J_{n-1, k(n-1)}\right]\left[D^{\prime}+J_{k(n-1), n-1}\right] \\
& =D D^{\prime}+J_{n-1, k(n-1)} D^{\prime}+D J_{k(n-1), n-1}+k(n-1) J_{n-1} \\
& =D D^{\prime}-k J_{n-1}-k J_{n-1}+k(n-1) J_{n-1} \\
& =D D^{\prime}+k(n-3) J_{n-1} .
\end{aligned}
$$

Let $r_{i}^{(z) \prime}$ be the reverse of row $i$ of $D_{z}$. Then, due to the cyclic construction of $D_{z}$, column $n-i$ of $D_{z}$ is $c_{n-i}^{(z)}=r_{i}^{(z)}$. Also, element $(p, q)$ of $D_{z} D_{z}^{\prime}$ is $r_{p}^{(z) \prime} r_{q}^{(z)}=c_{n-p}^{(z) \prime} c_{n-q}^{(z)}$. Writing $d=\left[D_{1}, \ldots, D_{k}\right]$,

$$
4 N N^{\prime}=\sum_{i=1}^{k} D_{i} D_{i}^{\prime}+k(n-3) J_{n-1} .
$$


Then since $\lambda_{p q}$ is the $(p, q)$ th element of $N N^{\prime}$, we have

$$
\begin{aligned}
4 \lambda_{p q}-k(n-3) & =\sum_{z=1}^{k} r_{p}^{(z) \prime} r_{q}^{(z)} \\
& =\sum_{z=1}^{k} c_{n-p}^{(z) \prime} c_{n-q}^{(z)} \\
& =\sum_{z=1}^{k}\left(s_{n-p, n-q}^{(z)}-1\right)
\end{aligned}
$$

and the result follows.

\section{Compatibility of circulant designs}

In order to achieve $E s^{2}$-optimal supersaturated designs by concatenating cyclic saturated designs, we look at the properties of the designs in terms of their autocorrelation functions as follows. Sequences $A_{i}, i=1,2, \ldots, k$, of identical length $\ell$ are called compatible if the sum of their periodic autocorrelations is a constant, say $\gamma$, except for the 0 -th term. That is,

$$
\sum_{i=1}^{k} P_{A_{i}}(x)=\gamma, \quad \text { for all } x=1,2, \ldots,[\ell / 2] .
$$

We say that such sequences have constant periodic autocorrelation sum. If $A_{1}, A_{2}, \ldots, A_{k}$ are compatible so that (4.1) holds, and if $D_{i}$ is a circulant matrix with first row given by the elements of $A_{i}$, then

$$
\sum_{i=1}^{k} D_{i} D_{i}^{\prime}=(k \ell-\gamma) I_{\ell}+\gamma J_{\ell}
$$

where $I_{\ell}$ is the identity matrix of order $\ell$ and $J_{\ell}$ is a square matrix of order $\ell$ with all its entries equal to one. Where there is no confusion, we will use the same symbols to represent sequences and their corresponding circulant matrices.

The following Theorem shows the link between the compatibility of sequences of length $\ell=n-1$ and designs that attain bound (2.2).

Theorem 4.1. Let $T$ be as described in Theorem 3.2. The following are equivalent:

(a) The design $T$ is an $E s^{2}$-optimal $k$-circulant mean-orthogonal supersaturated design with $n=2 t$ runs and $m=(2 t-1) k$ factors, satisfying bound (2.2).

(b) $\sum_{i=1}^{k} P_{G_{i}}(q)=\gamma$, where $\gamma=-k$, for all $q=1,2, \ldots, t-1$.

Proof. The proof follows from Result 3.1 together with Theorem 2 in Georgiou [12].

We note that Theorem 4.1 provides an alternative proof of Result 3.3 (originally proved by Cheng, 1997, Section 2) since, from Result $3.2 N=\left[N_{1}, \ldots, N_{k}\right]$ and $N_{i}=\left[D_{i}+J_{n-1}\right] / 2$, so 


$$
\begin{aligned}
N N^{\prime} & =\sum_{i=1}^{k} N_{i} N_{i}^{\prime} \\
& =\sum_{i=1}^{k} D_{i} D_{i}^{\prime}+\sum_{i=1}^{k} D_{i} J_{n-1}+\sum_{i=1}^{k} J_{n-1} D_{i}^{\prime}+k(n-1) J_{n-1} \\
& =\frac{k n}{4} I_{n-1}+\frac{k(n-4)}{4} J_{n-1}
\end{aligned}
$$

which is the concurrence matrix for a BIBD with parameter $\lambda=k(n-4) / 4$. Thus, the balanced incomplete block design of Example 3.1 has $\lambda=3(12-4) / 4=6$. Theorem 4.2 gives a formula for $E s^{2}$-efficiency of a $k$-circulant design when the corresponding periodic autocorrelation sequences are not compatible.

Theorem 4.2. Let $T$ be a mean-orthogonal supersaturated design as described in Theorem 3.2, with the property that $\sum_{i=1}^{k} P_{G_{i}}(q) \neq \gamma$ for at least one value of $q=1,2, \ldots, t-1$, then the value of $E s^{2}$ for $T$ is

$$
E s^{2}=\frac{n m(m-n)+(n-1)\left(\sum_{j=1}^{n-2} \gamma_{j}^{2}+2 k^{2}\right)}{m(m-1)}
$$

where $\gamma_{j}=\sum_{z=1}^{k} P_{G_{z}}(j)$ and $m=k(n-1)$.

Proof. By definition, since $T$ is a $k$-circulant mean-orthogonal supersaturated design, the row sum of each of its first $n-1$ rows is equal to $-k$ (see Section 3.1). Then from (3.2),

$$
T T^{\prime}=\left[\begin{array}{cc}
\sum_{z=1}^{k} D_{z} D_{z}^{\prime} & -k \mathbf{1}_{(n-1)} \\
-k \mathbf{1}_{(n-1)} & k(n-1)
\end{array}\right]
$$

where $\left[D_{z}^{\prime}, \mathbf{1}\right]^{\prime}$ is a 1 -circulant design with first row $G_{z}=\left(g_{1}^{(z)}, g_{2}^{(z)}, \ldots, g_{n-1}^{(z)}\right), z=1,2, \ldots, k$. Consider the $(u, v)$ th element, $d_{u, v}^{(z)}$, of $D_{z} D_{z}^{\prime}$ and let $x=|u-v|$. Then $d_{u, v}^{(z)}=\sum_{j=1}^{n-1} g_{j}^{(z)} g_{j+x}^{(z)}$. So, the $(u, v)$ th element of $\sum_{i=1}^{k} D_{z} D_{z}^{\prime}$ is

$$
\sum_{z=1}^{k} D_{z} D_{z}^{\prime}=\sum_{z=1}^{k} \sum_{i=0}^{n-2} g_{i+1}^{(z)} g_{i+1+x}^{(z)}=\sum_{z=1}^{k} P_{G_{z}}(x)=\gamma_{x} .
$$

Note that $\gamma_{0}=k(n-1)=m$, then

$$
T T^{\prime}=\left[\begin{array}{ccccccc}
m & \gamma_{1} & \gamma_{2} & \cdots & \cdots & \gamma_{n-2} & -k \\
\gamma_{1} & m & \gamma_{1} & \ddots & & \vdots & \vdots \\
\gamma_{2} & \gamma_{1} & m & \ddots & \ddots & \vdots & \vdots \\
\vdots & \ddots & \ddots & \ddots & \gamma_{1} & \gamma_{2} & -k \\
\vdots & & \ddots & \gamma_{1} & m & \gamma_{1} & -k \\
\gamma_{n-2} & \cdots & \cdots & \gamma_{2} & \gamma_{1} & m & -k \\
-k & -k & \cdots & \cdots & -k & -k & m
\end{array}\right]
$$


Since, from (3.1), $\gamma_{x}=\gamma_{(n-1)-x}$, each of the first $n-1$ diagonal elements of $\left(T T^{\prime}\right)\left(T T^{\prime}\right)$ is $m^{2}+k^{2}+\sum_{i=1}^{n-2} \gamma_{i}^{2}$. The $n$th diagonal element is $k^{2}(n-1)+m^{2}$. Now, by (2.1), $E s^{2}$ may be written

$$
\begin{aligned}
E s^{2}=\sum_{i \neq j=1}^{m} s_{i j}^{2} / m(m-1) & =\sum_{i, j=1}^{m}\left(s_{i j}^{2}-m n^{2}\right) / m(m-1) \\
& =\left(\operatorname{trace}\left(T^{\prime} T T^{\prime} T\right)-m n^{2}\right) / m(m-1) \\
& =\left(\operatorname{trace}\left(T T^{\prime} T T^{\prime}\right)-m n^{2}\right) / m(m-1) \\
& =\frac{n m^{2}+(n-1) \sum_{1}^{n-2} \gamma_{i}^{2}+2(n-1) k^{2}-m n^{2}}{m(m-1)} \\
& =\frac{n m(m-n)+(n-1)\left(\sum_{i=1}^{n-2} \gamma_{i}^{2}+2 k^{2}\right)}{m(m-1)}
\end{aligned}
$$

In the following example, we illustrate the construction of $E s^{2}$-optimal and near-optimal $k$-circulant supersaturated designs using the information from the autocorrelation function.

Example 4.1. Consider the construction of $E\left(s^{2}\right)$-optimal and near-optimal 10-run supersaturated designs using Theorems 3.1 and 4.1 with $n=2 t=10$ and $m=18,27,36,45$ factors. There are in total $9^{-1}\left(\begin{array}{c}9 \\ 5\end{array}\right)=14$ distinct generators of length 9 , where "distinct" means that the generated designs have no duplicated columns. Table 4.1 displays the 14 generators together with their periodic autocorrelation functions $P_{G_{i}}(q)$ for $q=1,2,3$, and 4 .

Consider the generators $G_{2}, G_{3}, G_{4}, G_{9}$ and $G_{11}$ and their respective 1-circulant $10 \times 9$ design matrices $T_{2}, T_{3}, T_{4}, T_{9}$ and $T_{11}$. For example, $G_{4}$ will produce

$$
T_{4}=\left(\begin{array}{l}
+++---+-- \\
-+++---+- \\
--+++---+ \\
+--+++-- \\
-+--+++-- \\
--+--+++- \\
---+--+++ \\
+---+--++ \\
++---+--+ \\
++++++++
\end{array}\right) \quad \text { with } P_{G_{4}}(1)=1, P_{G_{4}}(2)=-3, P_{G_{4}}(3)=-3, P_{G_{4}}(4)=1
$$

The 10-run design with $m=18$ factors, $\left[T_{2}, T_{11}\right]$, is $E\left(s^{2}\right)$-optimal since $\sum_{i=1}^{k=2} P_{G_{i}}(q)=-k$ for all $q=1,2,3,4$. Similarly for $m=36$, the design $\left[T_{2}, T_{3}, T_{9}, T_{11}\right]$ has $\sum P_{G}(q)=-4$ for $q=1,2,3,4$. There are no 10-run $E\left(s^{2}\right)$-optimal designs for $m=27$ or $m=45$ using cyclic generators. Out of the $\left(\begin{array}{c}14 \\ 3\end{array}\right)=364$ possible designs, there are 69 which have $E\left(s^{2}\right)=8.10$. Using equation (2.3) we obtain the lower bound of 7.92 which implies that all these designs are $97.7 \%$ efficient. One such design is $\left[T_{2}, T_{3}, T_{11}\right]$. The two designs $\left[T_{2}, T_{3}, T_{4}, T_{9}, T_{11}\right]$ and $\left[T_{1}, T_{3}, T_{10}, T_{12}, T_{14}\right]$ with $m=45$ factors are near $E s^{2}$-optimal designs with $E\left(s^{2}\right)=9.23$. Comparing this value to the lower bound we obtain $99.3 \%$ efficiency. It is interesting to note very different autocorrelation function values in Table 4.1 for $T_{i}$ 's which comprise these two designs. 
Table 4.1. Cyclic generators and their periodic autocorrelation functions

\begin{tabular}{|r|c|rrrr|}
\hline & & \multicolumn{4}{c}{$q$} \\
\hline & $G_{i}$ & 1 & 2 & 3 & 4 \\
\hline 1 & ++++----- & 5 & 1 & -3 & -7 \\
2 & +++-+---- & 1 & 1 & -3 & -3 \\
3 & +++--+--- & 1 & -3 & -3 & 1 \\
4 & +++---+-- & 1 & -3 & -3 & 1 \\
5 & +++----+- & 1 & 1 & -3 & -3 \\
6 & ++-++---- & 1 & -3 & 1 & -3 \\
7 & ++-+-+--- & -3 & 1 & -3 & 1 \\
8 & ++-+--+-- & -3 & -3 & 5 & -3 \\
9 & ++-+---+- & -3 & 1 & 1 & -3 \\
10 & ++--++--- & 1 & -7 & -3 & 5 \\
11 & ++--+-+-- & -3 & -3 & 1 & 1 \\
12 & ++--+--+- & -3 & -3 & 5 & -3 \\
13 & ++---+-+- & -3 & 1 & -3 & 1 \\
14 & +-+-+-+-- & -7 & 5 & -3 & 1 \\
\hline
\end{tabular}

Table 4.2. Equivalent generators for the designs with $n=10$ and $m=9 i, i \leq 7$

\begin{tabular}{|c|c|}
\hline Eskridge, Gilmour, Mead, Butler and Travnicek(2004) & From Table 4.1 \\
\hline$(0124)$ & $G_{5}$ \\
$(0125)(0137)$ & $G_{4} G_{9}$ \\
$(0134)(0135)$ & $G_{6} G_{13}$ \\
$(0124)(0146)$ & $G_{5} G_{11}$ \\
\hline
\end{tabular}

Bulutoglu and Cheng (2004) gave the complete set of solutions for 10-run supersaturated designs attaining bound (2.2) and they showed that they exist only for $m=18 t$ factors with $t=1, \ldots, 6$. Eskridge, Gilmour, Mead, Butler and Travnicek (2004) provided seven generators for cyclic incomplete block designs that produce $E s^{2}$ optimal (for $k$ even) or near optimal (for $k$ odd) supersaturated designs with $n=10$ runs and $m=9 i, i \leq 7$. Table 4.2 shows the seven generators provided by Eskridge, Gilmour, Mead, Butler and Travnicek (2004) and their equivalents from Table 4.1.

In Table 4.3, we present new $E s^{2}$-optimal supersaturated designs with $n$ runs $(10 \leq n \leq$ 26) and $m=k(n-1)$ factors for and their properties. We enumerate the elements of the cyclic generator and we present them in column 'Generators' using the incomplete block design notation. The second column of Table 4.3, labelled $f_{h}$ shows the frequency with which each value of $s_{i j}^{2}=h$ occurs in $T^{\prime} T$ for every integer $h$ for which the frequency is non-zero. The third column gives the maximum value $r_{\text {max }}$ of $s_{i j}^{2} / n$. Table 4.4 lists further new supersaturated designs with $k$ odd and which are at least $98.5 \%$ efficient; that is, whose $E s^{2}$ values are at least 0.985 of the corresponding lower bounds. The value of $E s^{2}$, the lower bound (2.3), and the efficiency $L B / E s^{2}$ is given for each design in columns 5-7 of the table. 
Table 4.3. Generators and properties for new $E s^{2}$-optimal supersaturated designs with $n$ runs and $m$ factors

\begin{tabular}{|c|c|c|c|c|}
\hline$n$ & $m$ & $f_{h}$ & $r_{\max }$ & Generators \\
\hline 10 & 90 & $3240_{2}, 765_{6}$ & 0.6 & $G_{2}-G_{9}, G_{11}, G_{13}$. \\
\hline 10 & 108 & $4640_{2}, 1134_{6}$ & 0.6 & $G_{1}-G_{5}, G_{7}-G_{12}, G_{14}$ \\
\hline 10 & 126 & $6300_{2}, 1575_{6}$ & 0.6 & $G_{1}-G_{14}$ \\
\hline 12 & 77 & $1144_{0}, 1683_{4}, 99_{8}$ & 0.667 & $\begin{array}{l}(0,1,4,7,9) ; \quad(1,5,6,8,10) ; \quad(0,2,3,7,10) ; \quad(0,3,7,8,9) \\
(0,1,3,5,6) ;(3,5,6,7,8) ;(0,1,6,7,8) .\end{array}$ \\
\hline 12 & 88 & $1452_{0}, 2244_{4}, 132_{8}$ & 0.667 & $\begin{array}{l}(0,1,6,7,9) ; \quad(1,5,7,8,9) ; \quad(0,2,3,6,10) ; \quad(0,3,5,6,7) ; \\
(0,3,5,8,9) ;(0,1,2,4,10) ;(0,2,4,6,7) ;(0,5,6,9,10) .\end{array}$ \\
\hline 12 & 99 & $1947_{0}, 2684_{4}, 220_{8}$ & 0.667 & $\begin{array}{llll}(0,1,5,6,7) ; & (1,5,6,8,10) ; & (0,2,3,6,8) ; & (0,3,4,6,7) ; \\
(0,1,2,8,10) ; & (0,1,2,4,5) ; & (0,2,5,9,10) ; & (0,2,5,6,9) ; \\
(0,2,3,5,6) & & & \end{array}$ \\
\hline 12 & 110 & $2431_{0}, 3267_{4}, 297_{8}$ & 0.667 & $\begin{array}{llll}(0,1,2,4,9) ; & (1,4,6,7,8) ; & (0,1,4,7,10) ; & (0,2,6,7,9) \\
(0,1,2,3,7) ; & (0,1,2,7,8) ; & (0,1,2,4,5) ; & (0,2,5,7,10) \\
(0,2,5,6,9) ; & (0,2,3,5,6) & & \end{array}$ \\
\hline 14 & 130 & $6032_{2}, 2262_{6}, 91_{10}$ & 0.714 & $\begin{array}{l}(0,1,2,4,6,8) ; \quad(2,4,5,6,10,11) ; \quad(0,3,5,6,9,12) ; \\
(0,3,5,7,10,11) ;(0,1,5,7,8,12) ;(0,1,2,4,5,9) ;(1,2,4,5,6,11) ; \\
(0,2,3,5,6,7) ;(0,2,3,5,8,11) ;(0,7,8,10,11,12) ;\end{array}$ \\
\hline 16 & 45 & $360_{0}, 600_{4}, 30_{8}$ & 0.5 & $(0,1,2,3,7,8,13) ;(0,1,5,7,9,10,13) ;(0,3,4,6,7,8,10)$ \\
\hline 16 & 60 & $510_{0}, 1200_{4}, 60_{8}$ & 0.5 & $\begin{array}{l}(0,1,2,5,7,11,13) ; \quad(0,1,3,5,8,11,12) ; \quad(0,1,2,4,5,7,14) ; \\
(3,4,5,9,10,12,13) .\end{array}$ \\
\hline 16 & 75 & $870_{0}, 1740_{4}, 165_{8}$ & 0.5 & $\begin{array}{l}(0,1,2,6,8,11,12) ; \quad(0,1,3,5,9,10,12) ; \quad(0,4,6,7,11,12,14) ; \\
(1,7,8,9,11,12,14) ;(0,1,2,3,4,6,9) .\end{array}$ \\
\hline 16 & 90 & $1350_{0}, 2340_{4}, 315_{8}$ & 0.5 & $\begin{array}{l}(0,5,7,8,10,12,13) ; \quad(1,3,5,6,9,12,14) ; \quad(0,1,2,5,6,9,12) ; \\
(0,5,6,9,12,13,14) ;(1,3,7,8,12,13,14) ;(0,1,2,6,12,13,14) .\end{array}$ \\
\hline 16 & 105 & $1860_{0}, 3120_{4}, 480_{8}$ & 0.5 & $\begin{array}{l}(0,1,2,5,7,9,10) ;(5,6,9,10,12,13,14) ; \quad(0,2,7,9,11,13,14) ; \\
(3,6,8,9,11,13,14) ; \quad(5,7,8,11,12,13,14) ; \\
(3,7,8,10,12,13,14) ;(2,7,8,10,11,13,14) .\end{array}$ \\
\hline 16 & 120 & $2400_{0}, 4080_{4}, 660_{8}$ & 0.5 & $\begin{array}{l}(0,1,2,5,7,8,11) ; \quad(5,6,8,9,12,13,14) ; \quad(0,2,7,10,11,12,14) ; \\
(3,6,8,11,12,13,14) ; \quad(0,1,2,4,6,8,13) ; \quad(0,2,5,6,8,11,13) ; \\
(0,2,3,6,12,13,14) ;(2,3,5,6,7,12,13) .\end{array}$ \\
\hline 16 & 135 & $3420_{0}, 4620_{4}, 1005_{8}$ & 0.5 & $\begin{array}{lll}(0,1,2,4,6,9,13) ; \quad(0,2,5,6,10,12,13) ; & (0,2,3,6,7,9,10) ; \\
(2,3,5,6,10,11,12) ; \quad(0,1,2,4,5,6,12) ; & (3,4,5,6,7,10,13) ; \\
(0,1,3,5,6,7,10) ;(0,3,4,5,9,11,13) ;(0,1,3,4,5,10,13)\end{array}$ \\
\hline 18 & 68 & $1530_{2}, 768_{6}$ & 0.333 & $\begin{array}{l}(0,1,5,6,12,13,14,15) ; \\
(0,1,5,8,10,11,14,16) ;(2,6,8,11,12,13,15,16) .\end{array}$ \\
\hline 18 & 102 & $3349_{2}, 1734_{6}, 68_{10}$ & 0.556 & $\begin{array}{lr}(0,2,3,4,6,7,9,16) ; & (0,3,6,8,9,10,15,16) \\
(0,2,3,6,7,11,12,16) ; & (0,1,3,5,6,7,12,15) ; \\
(0,1,2,3,5,9,11,15) ;(0,2,6,9,10,11,12,15)\end{array}$ \\
\hline 20 & 76 & $855_{0}, 1710_{4}, 285_{8}$ & 0.4 & $\begin{array}{l}(0,1,2,5,6,8,13,15,18) ; \quad(4,5,7,8,10,12,14,16,17) \\
(0,1,2,5,6,11,13,16,17) ;(4,5,8,12,13,14,15,16,18) .\end{array}$ \\
\hline 20 & 95 & $1254_{0}, 2698_{4}, 513_{8}$ & 0.4 & $\begin{array}{lr}(0,1,3,5,6,7,9,10,17) ; & (3,5,6,10,11,12,13,16,18) \\
(0,5,6,10,14,15,16,17,18) ; & (0,1,2,5,6,9,11,14,17) ; \\
(4,5,7,8,11,12,14,16,18) . & \end{array}$ \\
\hline
\end{tabular}




\begin{tabular}{|c|c|c|c|c|}
\hline$n$ & $m$ & $f_{h}$ & $r_{\max }$ & Generators \\
\hline 20 & 114 & $2109_{0}, 3401_{4}, 931_{8}$ & 0.4 & $\begin{array}{lr}(0,1,3,5,8,14,16,17,18) ; & (2,4,5,7,9,13,14,15,17) ; \\
(0,2,4,7,9,10,13,14,18) ; & (0,1,3,5,6,7,10,13,14) ; \\
(3,5,6,9,10,11,12,13,18) ; & (0,5,6,9,13,14,16,17,18)\end{array}$ \\
\hline 20 & 133 & $2964_{0}, 4427_{4}, 1387_{8}$ & 0.4 & $\begin{array}{lll}(0,1,5,7,8,13,15,16,18) ; & (0,3,5,9,11,15,16,17,18) ; \\
(0,2,3,10,11,12,13,17,18) ; & (4,6,7,8,10,11,13,14,16) ; \\
(0,1,4,5,7,9,12,14,16) ; & (3,4,7,9,10,13,15,16,18) ; \\
(0,1,6,10,11,14,15,16,18) & \end{array}$ \\
\hline 22 & 84 & $2058_{2}, 1407_{6}, 21_{10}$ & 0.454 & $\begin{array}{l}(0,1,6,8,9,12,14,15,18,19) \\
(1,4,8,12,13,14,15,16,17,18) \\
(0,2,5,7,9,10,11,13,18,19) \\
(2,4,7,11,12,13,14,17,18,19)\end{array}$ \\
\hline 22 & 126 & $4683_{2}, 2898_{6}, 294_{10}$ & 0.454 & $\begin{array}{l}(0,1,3,4,6,7,10,12,16,20) ; \quad(0,3,4,5,6,8,10,18,19,20) ; \\
(0,3,7,9,10,11,12,13,19,20) ; \\
(0,2,7,10,12,14,15,17,19,20) \\
(1,5,6,9,10,12,14,15,19,20) \\
(0,3,6,7,10,12,13,14,18,19)\end{array}$ \\
\hline 24 & 69 & $621_{0}, 1472_{4}, 253_{8}$ & 0.333 & $\begin{array}{l}(0,7,8,11,12,16,17,18,19,21,22) \\
(1,5,6,8,11,12,14,17,20,21,22) \\
(0,7,9,10,12,14,15,16,18,20,22)\end{array}$ \\
\hline 24 & 92 & $943_{0}, 2668_{4}, 575_{8}$ & 0.333 & $\begin{array}{l}(0,3,6,7,10,14,16,17,18,19,21) \\
(2,5,8,10,12,13,14,19,20,21,22) \\
(0,7,8,9,10,12,13,14,15,18,19) \\
(1,5,6,9,11,12,14,18,19,20,22)\end{array}$ \\
\hline 24 & 115 & $2346_{0}, 2852_{4}, 1357_{8}$ & 0.333 & $\begin{array}{l}(0,3,6,7,12,17,18,19,20,21,22) \\
(2,5,8,9,11,12,13,14,15,20,22) \\
(0,1,6,7,9,14,15,17,19,20,22) \\
(0,1,3,4,5,9,10,12,14,16,19) \\
(3,5,6,9,10,14,16,18,20,21,22)\end{array}$ \\
\hline 24 & 138 & $3082_{0}, 4393_{4}, 1955_{8}, 23_{12}$ & 0.5 & $\begin{array}{l}(0,1,3,7,8,9,12,17,19,20,22) ; \\
(2,3,4,5,9,11,14,15,18,20,21) ; \\
(0,4,11,12,13,14,15,16,19,20,21) ; \\
(0,3,6,7,8,10,11,13,15,16,20) ; \\
(0,2,6,8,10,11,14,15,19,20,21) ; \\
(1,5,6,7,8,10,11,13,19,21,22) .\end{array}$ \\
\hline 24 & 161 & $4278_{0}, 5865_{4}, 2622_{8}, 115_{12}$ & 0.5 & $\begin{array}{l}(0,2,3,5,6,7,10,11,15,21,22) \\
(2,4,5,7,10,12,13,14,15,16,21) \\
(2,3,4,9,10,14,15,16,17,18,20) \\
(4,5,9,10,12,14,16,17,18,19,21) \\
(0,3,6,7,8,10,11,13,17,20,22) \\
(0,2,6,8,10,11,13,14,16,17,21) \\
(1,5,6,7,8,10,11,14,16,20,21)\end{array}$ \\
\hline 26 & 50 & $850_{2}, 375_{6}$ & 0.231 & $\begin{array}{l}(0,2,6,7,9,12,14,17,18,21,23,24) \\
(0,8,10,12,13,16,17,18,21,22,23,24)\end{array}$ \\
\hline 26 & 100 & $2650_{2}, 2175_{6}, 125_{10}$ & 0.385 & $\begin{array}{l}(0,1,3,10,11,13,15,16,18,19,23,24) \\
(1,3,4,5,7,9,10,11,15,17,18,21) \\
(0,3,4,5,6,10,13,19,20,22,23,24) \\
(2,3,6,7,9,12,14,16,18,19,20,23)\end{array}$ \\
\hline
\end{tabular}


Table 4.4.Generators and properties for new near-optimal supersaturated designs with $n$ runs and $m=k(n-1)$ factors, with $k$ odd.

\begin{tabular}{|c|c|c|c|c|c|c|c|}
\hline$n$ & $m$ & $f_{h}$ & $r_{\max }$ & $E s^{2}$ & $L B$ & Eff & Generators \\
\hline 10 & 81 & $2628_{2}, 612_{6}$ & 0.6 & 10.04 & 10.02 & $99.8 \%$ & $G_{2}-G_{9}, G_{11}$ \\
\hline 10 & 99 & $3906_{2}, 945_{6}$ & 0.6 & 10.23 & 10.22 & $99.9 \%$ & $G_{1}-G_{3}, G_{5}, G_{7}-G_{13}$ \\
\hline 10 & 117 & $5436_{2}, 1350_{6}$ & 0.6 & 10.37 & 10.36 & $99.9 \%$ & $G_{1}, G_{3}-G_{14}$ \\
\hline 14 & 39 & $585_{2}, 156_{6}$ & 0.429 & 10.74 & 10.56 & $98.4 \%$ & $\begin{array}{l}(0,5,6,8,9,11) \\
(2,6,7,8,11,12) ;(5,6,7,9,11,12)\end{array}$ \\
\hline 14 & 65 & $1534_{2}, 546_{6}$ & 0.429 & 12.4 & 12.35 & $99.5 \%$ & $\begin{array}{ll}(0,2,3,6,11,12) ; & (0,1,4,6,9,12) ; \\
(0,2,4,6,9,10) ; & (0,1,2,7,11,12) ; \\
(0,2,4,6,7,12) ; & \end{array}$ \\
\hline 14 & 91 & $2925_{2}, 1170_{6}$ & 0.429 & 13.14 & 13.11 & $99.8 \%$ & $\begin{array}{ll}(0,3,5,6,9,12) ; & (1,4,6,9,10,11) ; \\
(0,4,6,7,11,12) ; & (0,1,4,10,11,12) ; \\
(1,3,4,5,7,9) ; & (0,3,5,7,10,11) ; \\
(0,1,2,4,5,9) & \end{array}$ \\
\hline 18 & 51 & $901_{2}, 374_{6}$ & 0.333 & 13.39 & 13.19 & $98.5 \%$ & $\begin{array}{l}(0,3,6,8,9,12,13,15) \\
(2,3,4,9,10,11,13,14) \\
(2,5,6,7,9,10,11,13)\end{array}$ \\
\hline 18 & 85 & $2295_{2}, 1258_{6}, 17_{10}$ & 0.556 & 15.73 & 15.51 & $98.6 \%$ & $\begin{array}{l}(0,2,3,6,10,12,14,15) ; \\
(5,6,9,10,13,14,15,16) ; \\
(1,5,6,8,12,13,14,16) ; \\
(3,8,10,11,12,13,14,15) ; \\
(1,2,4,9,10,13,15,16)\end{array}$ \\
\hline 18 & 119 & $4641_{2}, 2193_{6}, 187_{10}$ & 0.556 & 16.55 & 16.52 & $99.8 \%$ & $\begin{array}{l}(1,6,7,8,10,11,15,17) ; \\
(3,4,5,6,7,8,12,17) ; \\
(2,3,5,8,10,13,14,16) ; \\
(2,4,6,10,13,14,15,16) ; \\
(1,2,3,5,11,13,14,15) ; \\
(2,3,7,9,10,12,14,16) ; \\
(1,2,4,6,7,13,14,17)\end{array}$ \\
\hline 22 & 63 & $1218_{2}, 735_{6}$ & 0.273 & 16.04 & 15.85 & $98.8 \%$ & $\begin{array}{l}(0,3,4,6,7,9,10,16,18,20) \\
(3,6,9,10,11,12,14,18,19,20) \\
(1,5,10,11,12,13,15,17,18,20)\end{array}$ \\
\hline 26 & 75 & $1750_{2}, 900_{6}, 125_{10}$ & 0.385 & 18.70 & 18.50 & $98.9 \%$ & $\begin{array}{l}(3,6,7,8,12,14,15,17,19,20,21,23) ; \\
(4,6,7,8,10,11,12,14,16,19,20,21) ; \\
(0,1,4,7,10,12,14,15,19,20,21,22)\end{array}$ \\
\hline
\end{tabular}

\section{Acknowledgements}

This work was partly supported by grant SES-0437251 from the National Science Foundation. The authors would like to thank the referee for many helpful comments.

\section{References}

Abraham, B., Chipman, H., Vijayan K., 1999. Some risks in the construction and analysis of supersaturated designs. Technometrics, 41 (2), 135-141.

Beattie, S.D., Fong, D.K.H., Lin, D.K.J., 2002. A two-stage Bayesian model selection strategy for supersat- 
urated designs. Technometrics, 22 (1), 55-63.

Booth, K.H.V., Cox, D.R., 1962. Some systematic supersaturated designs. Technometrics, 4, 489-495.

Box, G.E.P., Meyer, R.D., 1986. An analysis for unreplicated fractional factorials. Technometrics, 28, $11-18$.

Bulutoglu, D.A., Cheng, C.S., 2004. Construction of $E\left(s^{2}\right)$-optimal supersaturated designs. Annals of Statistics, 32, 1662-1678.

Butler, N.A., Mead, R., Eskridge, K.M., Gilmour, S.G., 2001. A general method of constructing $E\left(s^{2}\right)$ optimal supersaturated designs. J. R. Statist. Soc., B, 63, 621-632.

Chen, J., Lin, D.K.J., 1998. On the identifiability of a supersaturated design. Journal of Statistical Planning and Inference, 72, 99-107.

Cheng, C.S., 1997. E $\left(s^{2}\right)$-optimal supersaturated designs. Statistica Sinica, 7, 929-939.

Chipman, H., Hamada, M., Wu, C.F.J., 1997. A Bayesian variable-selection approach for analyzing designed experiments with complex aliasing. Technometrics, 39, 372-381.

Das, A., Dey, A., Chan, L., Chatterjee, K. (2008). On $E\left(s^{2}\right)$-optimal supersaturated designs. Journal of Statistical Planning and Inference, 138, 3746-3757.

Eskridge, K.M., Gilmour, S.G., Mead, R., Butler, N.A., Travnicek, D.A., 2004. Large supersaturated designs. J. Stat. Comput. Simul., 74, 525-542.

Georgiou, S.D., 2008. On the construction of $E\left(s^{2}\right)$-optimal supersaturated designs, Metrika, 68, 189-198.

Geramita, A.V., Seberry, J., 1979. Orthogonal Designs: Quadratic forms and Hadamard Matrices. Marcel Dekker, New York-Basel.

Kelly, H.W., III, Voelkel, J.O., 2000. Asymptotic-power problems in the analysis of supersaturated designs. Statistics and Probability Letters, 47, 317-324.

Li, R., Lin, D.K.J., 2003. Analysis methods for supersaturated design: Some comparisons. Journal of Data Science, 1, 249-260.

Li, W., Wu, C.F.J., 1997. Columnwise-pairwise algorithms with applications to the construction of supersaturated designs. Technometrics, 39, 171-179.

Lin, D.K.J., 1993. A new class of supersaturated designs. Technometrics, 35, 28-31.

Lin, D.K.J., 1995. Generating systematic supersaturated designs. Technometrics, 37, 213-225.

Liu, Y., Dean, A.M., 2004. k-circulant supersaturated designs. Technometrics, 46, 32-43.

Liu, Y., Ruan, S., Dean, A.M., 2006. Construction and analysis of $E s^{2}$ efficient supersaturated designs. Journal of Statistical Planning and Inference, 137, 1516-1529.

Liu, M., Zhang, R., 2000. Construction of $E\left(s^{2}\right)$ optimal supersaturated designs using cyclic BIBDs. Journal of Statistical Planning and Inference, 91, 139-150.

Meyer, R.D., Wilkinson, R.G., 1998. Bayesian variable assessment. Communications in Statistics: Theory and Methods, 27, 2675-2705.

Nguyen, N.K., 1996. An algorithmic approach to constructing supersaturated designs. Technometrics, 38, 69-73.

Phoa, F., Pan, Y.-H., Xu, H., 2007. Analysis of Supersaturated Designs via Dantzig Selector. Technical Report, 2007010118, University of California at Los Angeles. http://repositories.cdlib.org/uclastat/papers/2007010118.

Plackett, R.L., Burman, J.P., 1946. The design of optimum multifactorial experiments. Biometrika, 33, 305-325.

Satterthwaite, F.E., 1959. Random balance experimentation (with discussions). Technometrics, 1, 111-137.

Ryan, K.J., Bulutoglu, D.A., 2007. $E s^{2}$-optimal supersaturated designs with good minimax properties. Journal of Statistical Planning and Inference, 137, 2250-2262.

Street, A.P., Street, D.J., 1987. Combinatorics of Experimental Design. Oxford Science Publications.

Tang, B., Wu, C.F.J., 1997. A method for constructing supersaturated designs and its $E s^{2}$ optimality. Canadian Journal of Statistics, 25, 191-201.

Wallis, W.D., Street, A.P., Seberry, J. Wallis, 1972. Combinatorics: Room Squares, Sum-Free Sets, Hadamard Matrices. Lecture Notes in Mathematics, Vol. 292, Springer-Verlag, Berlin, Heidelberg, New York.

Westfall, P.H., Young, S.S., Lin, D.K.J., 1998. Forward selection error control in the analysis of supersaturated designs. Statistica Sinica, 8, 101-117.

Wu, C.F.J., 1993. Construction of supersaturated designs through partially aliased interactions. Biometrika, $80,661-669$. 